\title{
Análise Fluidodinâmica da Região de Gaseificação de um Gaseificador de Leite Fluidizado
}

\section{Barbosa, K. R. M.}

Escola Politécnica de Pernambuco Universidade de Pernambuco 50.720-001 - Recife, Brasil

Katiamointeiro15@gmail.com

\author{
Silva, J. D. \\ Escola Politécnica de Pernambuco \\ Universidade de Pernambuco \\ 50.720-001 - Recife, Brasil
}

\footnotetext{
Resumo A geração de energia através da queima de biomassa é uma alternativa sustentável devido à produção de energia com baixa emissão de poluentes e por possui uma fonte renovável, como bagaço de cana e resíduos plásticos, possibilitado gerar um equilíbrio entre o consumo e produção de gás carbônico. A gaseificação em leito fluidizado é uma forma de aumentar a eficiência do uso energético da biomassa. A fluidização baseia-se fundamentalmente na circulação de sólidos juntamente com um fluido (gás ou líquido) impedindo a existência de gradientes de temperatura, de pontos muito ativos ou de regiões estagnadas no leito; proporcionando também um maior contato superficial entre sólido e fluido, favorecendo a transferência de massa e calor. A eficiência na utilização de um leito fluidizado depende em primeiro lugar do conhecimento da velocidade mínima de fluidização. Abaixo desta velocidade o leito não fluidiza; e muito acima dela, os sólidos são carregados para fora do leito. Será desenvolvido um conjunto de equações a fim de analisar a sensibilidade das frações volumétrica, velocidade e pressão na região de fluidodinâmica.
} 


\section{Introdução}

Os gaseificadores de leito fluidizado caracterizamse pela formação de um leito de biomassa em suspensão produzido por efeito do fluxo de ar forçado através de um distribuidor. É importante o bom contato entre a biomassa e o ar no leito fluidizado para que haja uma transferência de massa e energia com facilidade. A parte vital do leito fluidizado é sua alimentação de ar e combustíveis visto que sofre uma variação na quantidade de gás consumido pela turbina.

A principal vantagem dos gaseificadores de leito fluidizado sobre o de leito fixo consiste na distribuição uniforme da temperatura na região de gaseificação, pois o material inerte de baixa granulometria, que mediante a injeção de ar ou outro agente de gaseificação em pressões acima da atmosférica, entra em regime de fluidização. $\mathrm{O}$ estado dinâmico do leito inerte(alumina, areia) assegura uma mistura homogenia coma a biomassa alimentada e os gases quentes da combustão parcial (CENBIO,2002).

Os gases obtidos durante a gaseificação são provenientes de parte orgânica do combustível que se transforma principalmente em $\mathrm{CO}, \mathrm{H} 2$, e em menos proporções no $\mathrm{CH} 4$ e outros compostos hidrocarbonetos. Sendo constituído também por outros gases inertes e elementos indesejáveis, como alcatrão, metais alcalinos, particulados sólidos, sulfetos de hidrogênio, amônia. Estes componentes devem ser removidos, para que não danifiquem os equipamentos que irão utilizá-los. (NETO,2001).

A limpeza pode ocorrer à baixa temperatura, por exemplo, por meio de filtragem(que ocorre em torno de $200^{\circ} \mathrm{C}$ ) e lavagem, para remoção de particulados e condensáveis, após prévio resfriamento. A limpeza também pode ser realizada a média-alta temperatura $\left(350^{\circ}-400^{\circ} \mathrm{C}\right)$, para sua utilização em turbinas a gás e células a combustível. Em geral, essa limpeza a quente é feita com emprego de filtros cerâmicos[Macedo ET AL. 2006].

\section{Descrição do modelo matemático}

No desenvolvimento do modelo, foram consideradas algumas hipóteses:

1. os fluxos são unidimensionais;

2. A fase fluida é compressível

3. Todas as partículas têm a mesma dimensão

4. O movimento irregular e a colisão das partículas são ignoradas

5. A fricção sobre a parede é desprezada. Além de ser restrito a zona de fluidização como é mostrada na figura abaixo:

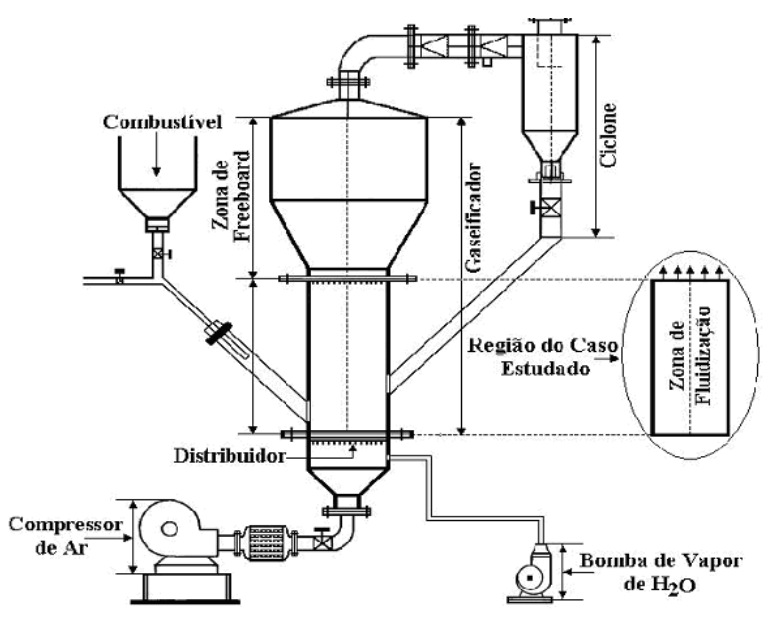

O fluxo de combustível não é fácil de manter estável. Dessa forma, os modelos fluidodinâmicos permitem elaborar os balanços de massa e momento para traçar estratégia operacional que possibilitam a quantificação de variáveis operacionais estáveis (Arvelakis et al., 200, Savage, 1998).

- Balanço de massa para a fase gasosa;

$$
\frac{\partial \xi_{g}}{\partial t}+V_{g} \frac{\partial \xi_{g}}{\delta z}=\left(1-\xi_{s}\right) \frac{g}{V_{g}}
$$

- Balanço de massa para a fase solida;

$$
\frac{\partial \xi_{s}}{\partial t}+V_{s} \frac{\partial \xi_{s}}{\delta z}=\left(1-\xi_{g}\right) \frac{g}{V_{s}}
$$

- Balanço de momento para a fase gasosa;

$$
\begin{aligned}
& \rho_{g}\left(\xi_{g} \frac{\partial V_{g}}{\partial t}+2 \xi_{g} V_{g} \frac{\partial V_{g}}{\partial z}\right)=-\rho_{g}\left(V_{g} \frac{\partial \xi_{g}}{\partial t}+V_{g}^{2} \frac{\partial \xi_{g}}{\partial z}\right) \\
& -\left(1-\xi_{s}\right) \frac{\partial P}{\partial z}-\xi_{g} \rho_{g} g-F_{s}
\end{aligned}
$$

- Balanço de momento para a fase sólida;

$$
\begin{aligned}
& \rho_{s}\left(\xi_{s} \frac{\partial V_{s}}{\partial t}+2 \xi_{s} V_{s} \frac{\partial V_{s}}{\partial z}\right)=-\rho_{s}\left(V_{s} \frac{\partial \xi_{s}}{\partial t}+V_{s}^{2} \frac{\partial \xi_{s}}{\partial z}\right) \\
& -\left(1-\xi_{g}\right) \frac{\partial P}{\partial z}-\xi_{s} \rho_{s} g-F_{s}
\end{aligned}
$$

A soma da Equação (3) com a Equação (4) resulta em uma única equação para a pressão, assim expressa: 


$$
\begin{aligned}
& -\frac{\partial P}{\partial z}=\rho_{s}\left[\left(\xi_{s} \frac{\partial V_{s}}{\partial t}+V_{s} \frac{\partial \xi_{s}}{\partial z}\right)+\left(\frac{\partial V_{s}}{\partial z} 2 \xi_{s} V_{s}+V_{s}^{2} \frac{\partial \xi_{s}}{\partial z}\right)\right]+ \\
& \rho_{g}\left[\left(\xi_{g} \frac{\partial V_{g}}{\partial t}+V_{g} \frac{\partial \xi_{g}}{\partial z}\right)+\left(\frac{\partial V_{g}}{\partial z} 2 \xi_{g} V_{g}+V_{g}^{2} \frac{\partial \xi_{g}}{\partial z}\right)\right] \\
& +\left(\rho_{g} \xi_{g}+\rho_{s} \xi_{s}\right) g
\end{aligned}
$$

\begin{tabular}{|l|l|}
\hline Condições iniciais & \multicolumn{1}{c|}{$\begin{array}{l}\text { Condições de } \\
\text { contorno }\end{array}$} \\
\hline$\left.\xi_{g}\right|_{t=0}=0$ & $\left.\xi_{g}\right|_{z=0^{+}}=\xi_{g, 0}$ \\
\hline$\left.\xi_{s}\right|_{t=0}=0$ & $\left.\xi_{s}\right|_{z=0^{+}}=\xi_{s, 0}$ \\
\hline$\left.V_{g}\right|_{t=0}=0$ & $\left.V_{g}\right|_{z=0^{+}}=V_{g, 0}$ \\
\hline$\left.V_{s}\right|_{t=0}=0$ & $\left.V_{s}\right|_{z=0^{+}}=V_{s, 0}$ \\
\hline & $\left.P\right|_{z=0^{+}}=P_{0}$ \\
\hline
\end{tabular}

Pelas condições iniciais e discretizando as condições de contorno depois ao reorganizar as equações de balanço de massa e momento para as fases gasosas e sólidas foi possíveis chegar às seguintes equações:

- Balanço de massa para a fase gasosa, discretizado;

$$
\frac{d \xi_{g}}{d t}=\left[1-\left(\xi_{s}\right)_{j}^{(k)}\right] \frac{g}{\left(V_{g}\right)_{j}^{(k)}}-\frac{2\left(V_{g}\right)_{j}^{(k)}}{\Delta z}\left[\xi_{g, 0}-\left(\xi_{g}\right)_{j}^{(k)}\right]
$$

- Balanço de massa para a fase sólida, discretizado;

$$
\frac{d \xi_{s}}{d t}=\left[1-\left(\xi_{g}\right)_{j}^{(k)}\right] \frac{g}{\left(V_{s}\right)_{j}^{(k)}}-\frac{2\left(V_{s}\right)_{j}^{(k)}}{\Delta z}\left[\xi_{s, 0}-\left(\xi_{s}\right)_{j}^{(k)}\right]
$$

- Balanço de momento para a fase gasosa, discretizado;

$$
\begin{aligned}
& -\frac{d V_{g}}{d t}=\frac{\left[1-\left(\xi_{s}\right)_{j}^{(k)}\right]}{\left(\xi_{g}\right)_{j}^{(k)}}\left\{g+\frac{2}{\rho_{g} \Delta z}\left[P_{0}-(P)_{j}^{(k)}\right]\right\}+ \\
& +\frac{4\left(V_{g}\right)_{j}^{(k)}}{\Delta z}\left[V_{g, 0}-\left(V_{g}\right)_{j}^{(k)}\right]+\frac{\left(F_{s}\right)_{j}^{(k)}}{\rho_{g}\left(\xi_{g}\right)_{j}^{(k)}}+g
\end{aligned}
$$

- Balanço de momento para a fase sólida, discretizado;

$$
\begin{aligned}
& -\frac{d V_{s}}{d t}=\frac{\left[1-\left(\xi_{g}\right)_{j}^{(k)}\right]}{\left(\xi_{s}\right)_{j}^{(k)}}\left\{g+\frac{2}{\rho_{s} \Delta z}\left[P_{0}-(P)_{j}^{(k)}\right]\right\}+ \\
& +\frac{4\left(V_{s}\right)_{j}^{(k)}}{\Delta z}\left[V_{s, 0}-\left(V_{s}\right)_{j}^{(k)}\right]+\frac{\left(F_{s}\right)_{j}^{(k)}}{\rho_{s}\left(\xi_{s}\right)_{j}^{(k)}}+g
\end{aligned}
$$

Nas quais as equações constitutivas discretizadas são dadas por:

$$
\begin{aligned}
& -\frac{d P}{d z}=\rho_{s}\left\{\begin{array}{l}
-g\left[1-\left(\xi_{g}\right)_{j}^{(k)}\right]-\frac{\left.21-\left(\xi_{g}\right)_{j}^{(k)}\right]}{\rho_{s} \Delta z}\left[P_{0}-(P)_{j}^{(k)}\right]-\frac{\left(F_{s}\right)_{j}^{(k)}}{\rho_{s}}+ \\
\frac{2\left(V_{s}\right)_{j}^{(k)}\left[\xi_{s, 0}-\left(\xi_{s}\right)_{j}^{(k)}\right]}{\Delta z}\left[1+\left(V_{s}\right)_{j}^{(k)}\right]
\end{array}\right\} \\
& +\rho_{g}\left\{\begin{array}{l}
-g\left[1-\left(\xi_{s}\right)_{j}^{(k)}\right]-\frac{2\left[1-\left(\xi_{s}\right)_{j}^{(k)}\right]}{\rho_{g} \Delta z}\left[P_{0}-(P)_{j}^{(k)}\right]-\frac{\left(F_{s} s_{j}^{(k)}\right.}{\rho_{g}}+ \\
\frac{2\left(V_{g}\right)_{j}^{(k)}\left[\xi_{g, 0}-\left(\xi_{g}\right)_{j}^{(k)}\right]}{\Delta z}\left[1+\left(V_{g}\right)_{j}^{(k)}\right]
\end{array}\right\}
\end{aligned}
$$

A equações: (1) a (4) são insuficiente para dar uma resposta específica do problema. Sendo necessário complementar com as seguintes equações:

$$
\begin{aligned}
& F_{s}=\frac{\left(\rho_{s}-\rho_{g}\right) g \varepsilon_{g}\left(1-\varepsilon_{s}\right)^{(1-n)}\left(v_{g}-v_{s}\right)}{v_{t}} \\
& v_{t}=\left(\frac{\rho_{s}-\rho_{g}}{\rho_{s}}\right) g \tau \quad ; \quad \tau=\frac{d_{p}^{2} \rho_{s}}{k \mu_{g}}
\end{aligned}
$$

\section{Metodologia numérica}

O sistema analisado consiste das equações fundamentais de balanço de massa para as fases gasosa e sólida, como também das equações de balanços de momento para as fases gasosas e sólidas e uma equação para a pressão obtido através da soma das equações de balanço de momento das fases gasosas e sólidas. Os conjuntos de equações desenvolvidos formam um sistema de equações parciais (EDPs) unidimensionais. O sistema de EDPs foi resolvido com a implementação do método das diferenças finitas implícito (MDFI) usando o esquema de Crank-Nicolson.

A partir das Equações (6) a (12), const constrói-se o SEA com equações em função de $(\mathrm{k}+1)$ e $(\mathrm{k})$. O SEA foi resolvido com a implementação do método Rang Kutta Gil. 


\section{Resultados e discussões}

Os parâmetros utilizados para a realização da simulação estão expressos abaixo:

\begin{tabular}{|l|c|}
\hline \multicolumn{1}{|c|}{ Parâmetros } & Unidade \\
\hline$\rho_{s}=1,21$ & $\mathrm{~kg} \cdot \mathrm{m}^{-3}$ \\
\hline$\rho_{g}=1150$ & $\mathrm{~kg} \cdot \mathrm{m}^{-3}$ \\
\hline$\mu_{g}=1,8 \times 10^{-5}$ & Pa.s \\
\hline$g=9,8$ & $\mathrm{~m} \cdot \mathrm{s}^{-2}$ \\
\hline$d_{g}=500$ & $\mu m$ \\
\hline$n=1,37$ & - \\
\hline$k=0,8$ & - \\
\hline$\Delta t=10^{-2}$ & $\mathrm{~s}$ \\
\hline
\end{tabular}

Figura - 1: Perfis da Fração volumétrica da fase gasosa na saída do gaseificador de leito fluidizado em contracorrente com comprimento de $1 \mathrm{~m}$

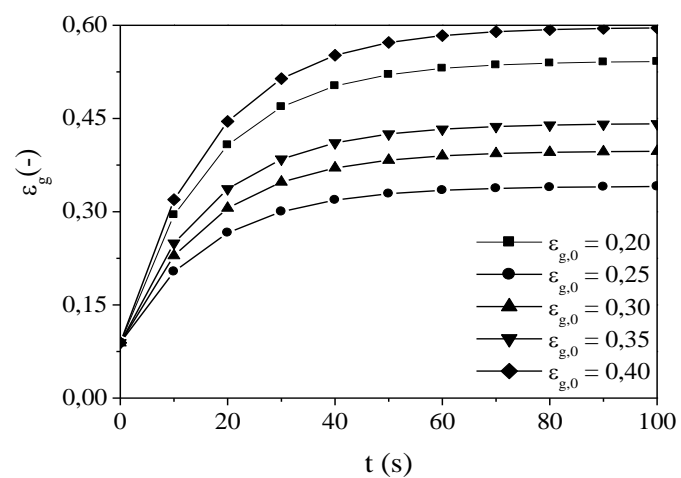

Figura - 2: Perfis da Fração volumétrica da fase sólida na saída do gaseificador de leito fluidizado em contracorrente com comprimento de $1 \mathrm{~m}$

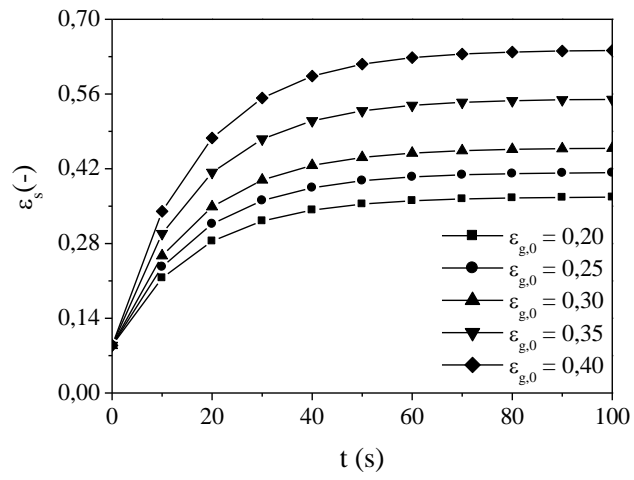

As figuras 1 e 2 mostram a evolução dinâmica das frações volumétricas $\xi_{g}$ e $\xi_{s}$ em $z=H$. Observa-se que houve um aumento destas frações com o aumento do parâmetro $\xi_{g, 0}$ na entrada do sistema. As frações volumétricas são vão encontrar um estado estacionário em torno de $\mathrm{t}=100$. As duas frações apresentaram um resultado bastante parecidos.

Figura - 3: Perfis da velocidade superficial da fase gasosa na saída do gaseificador de leito fluidizado borbulhante em contracorrente com comprimento de $1 \mathrm{~m}$

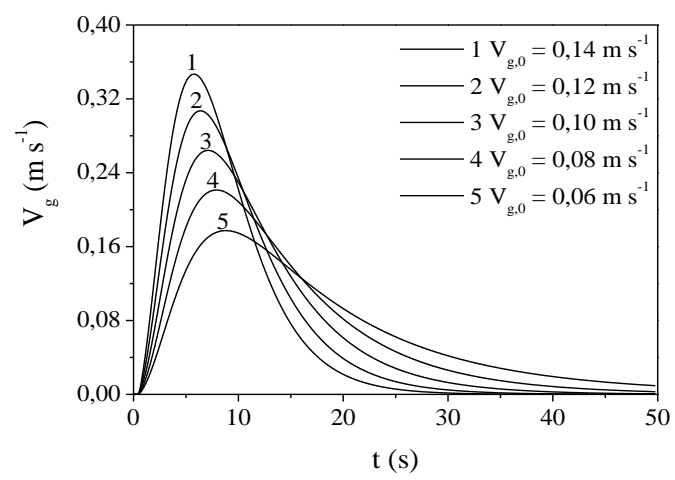

Figura - 4: Perfis da velocidade superficial da fase sólida na saída do gaseificador de leito fluidizado borbulhante em contracorrente com comprimento de $1 \mathrm{~m}$

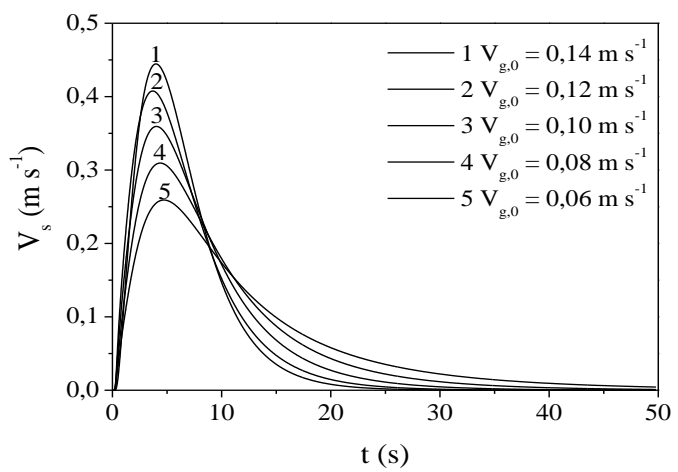

As figuras 3 e 4 mostram a evolução dinâmica das frações dinâmica das velocidades das fases gasosa e sólida $V_{g}$ e $V_{s}$ em $\mathrm{z}=\mathrm{H}$. Os gráficos mostram que tais frações também sofreram um aumento substancial com o aumento da variável $V_{g, 0}$ de entrada, porém até os 5 s a 8 s na fração $V_{g}$ e nos $4 \mathrm{~s}$ a $5 \mathrm{~s}$ na fração $V_{s}$ houve uma quedra até zero. Pode-se observar também que com o aumento da variável de entrada $V_{g, 0}$ a queda das frações $V_{g} \mathrm{e}_{V_{s}}$ foi mais rápida. 
Figura - 5: Perfis da queda de pressão no gaseificador de leito fluidizado borbulhante em contracorrente com comprimento de $1 \mathrm{~m}$

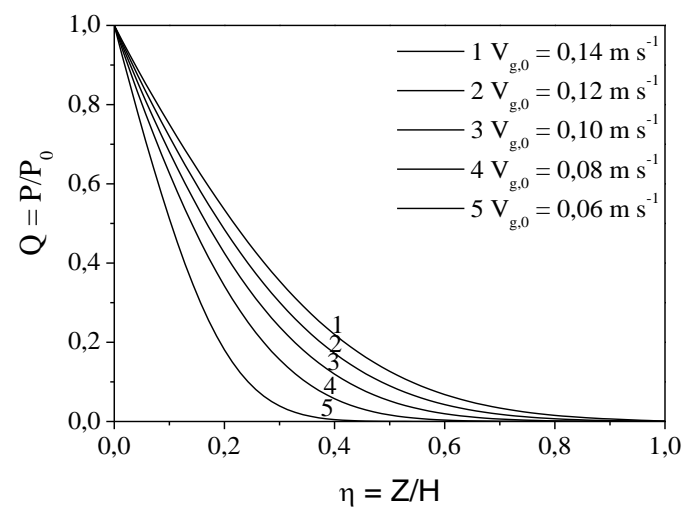

A figura 5 apresenta o campo de pressão ao longo da região de fluidização. Observa-se o efeito de velocidade $V_{g, 0}$ de entrada sobre a pressão no interior do LF. Mostrando no gráfico que a velocidade se apresentou proporcional ao aumento de pressão até zerar.

\section{Conclusão}

Esse trabalho pode-se entender como funciona um gaseificador de leito fluidizado, compreendendo suas vantagens a fim de desenvolver uma análise da sensibilidade das variáveis $\xi_{g} ; \xi_{s ;} V_{g ;} V_{s ;} p$ para a região específica de fluidização. Foi desenvolvida uma modelagem da qual foi empregada variáveis de entrada $\xi_{g, 0}$ e $V_{g, 0}$ mostrando sua influência sobre o processo gás-sólido a fim de ser consideradas no controle no processo desse equipamento. E não fica restrito o uso apenas dessas variáveis de entrada, as outras variáveis $\xi_{s, 0}$ e $V_{s, 0}$ também podem ser testadas.

\section{Agradecimentos}

Os autores deste artigo gostariam de agradecer ao CNPQ (Conselho Nacional de Desenvolvimento Científico e Tecnológico) pelo suporte financeiro dado. (Processo 48354 / 2010 / Projeto / Título: Análise Fluidodinâmica da Região de Gaseificação de um Gaseificador de Leito Fluidizado. / Edital CNPq 15 / 2010 - Universal).

\section{Nomenclatura}

Dp - Diâmetro das partículas, mFs - Força de interação entre as fases gasosa e sólida por unidade de volume, kg s$2 \mathrm{~m}-2$

g - Aceleração da gravidade, $\mathrm{m} \mathrm{s-2}$

H - Comprimento da zona de fluidização, m

k - Constante da Equação (9)

n - Constante da Equação (10)

$\mathrm{P}$ - Pressão, $\mathrm{Pa}$

P0 - Pressão inicial, Pa

$\mathrm{t}$ - Coordenada temporal, $\mathrm{s}$

$\mathrm{Vg}$ - Velocidade da fase gasosa, $\mathrm{m} \mathrm{s}-1$

$\mathrm{Vg}, 0$ - Velocidade da fase gasosa inicial, $\mathrm{m} \mathrm{s}-1$

Vs - Velocidade da fase sólida, m s-1

$\mathrm{Vt}$ - Velocidade terminal da partícula dolíquido, m s-1

z - Coordenada espacial, $m$

\section{Letras Gregas}

घg - Fração volumétrica da fase gasosa

घg,0 - Fração volumétrica da fase gasosa inicial

Es - Fração volumétrica da fase sólida

$\mu \mathrm{g}$ - Coeficiente de viscosidade da fase gasosa,Pa $\mathrm{s}$

$\rho g$ - Densidade da fase gasosa, $\mathrm{kg} \mathrm{m-3}$

$\rho$ - Densidade da fase sólida, $\mathrm{kg} \mathrm{m}-3$

$\tau$ - Fator de relaxação $(0 \leq \tau \leq 1)$ para a fase gasosa, $s$

Q - Pressão, adimensionalizada

$\Delta \mathrm{t}$ - Passo de tempo, $\mathrm{s}$

$\zeta$ - Comprimento da zona de fluidização, adimensionalizado

\section{Referências}

[1] NEIVA, A.C.B. Estudo de alimentadores de Bagaço Dissertação de Cana para Reatores Atmosféricos, de Mestrado, Faculdade de Engenharia Mecânica, UNICAMP. Campinas, 1998.

[2] SÁNCHEZ, C.G. Estudo da volatilização e da gaseificação de biomassa em leito fluidizado, Tese de Doutorado, Faculdade de Engenharia Mecânica, UNICAMP. Campinas, 1994. 
[3] ROSAL, A.G.C. Modelagem e simulação de um gaseificador de biomassa em leito fluidizado borbulhante para produção de energia, Tese de Doutorado, Universidade Federal de Pernambuco, UFPE. Recife, 2008.

[4] Prof. Dr. Marcello Nitz e Prof. Dr. Roberto Guardani Fluidização Gás-Sólido - Fundamentos e Avanços Escola de Engenharia Mauá, Centro Universitário do Instituto Mauá de Tecnologia e Departamento de Engenharia Química, Escola Politécnica da USP

[5] Bioetanol de cana-de-açúcar: energia para o desenvolvimento sustentável / organização BNDES e CGEE. - Rio de Janeiro: BNDES, 2008.

[6] Prof. GUALBERTO, L. Comparação entre tecnologias de gadeificação de biomassa existentes no brasil e no exterior e formação de recursos humanos na região norte. Cidade Universitária - São Paulo

[7] NETO, V.C Análise da cogeração de energia elétrica em ciclo combinado com gaseificação de biomassa de cana-de-açúcar e gás natural. Dissertação de Mestrado/ CPPE - UFRJ, 2001.

[8] COELHO, ST.,MARTINS, O.S.,SANTOS,S.M.A. Estado da arte da gaseificação. CENBIO, 2002.

[9] ARVELAKIS, P.; VOURLIOTIS, E.; KAKARAS E.; G. KOUKIOS, G., (2001),"'Eggect of leaching oh the ash behavior of wheat straw and olive residue during fluidized bed combustion". Biomass and Bioenergy, v.26,p459-470. 